\title{
Coupled Circuit-Interconnect Modeling and Simulation
}

\section{Luís Miguel Silveira}

Cadence European Laboratories/INESC, Dept. of Electrical and Computer Engineering, Instituto Superior Técnico

Rua Alves Redol, 9, 136, 1000 Lisboa, Portugal, Tel: 351-1-3100337, Fax: 351-1-3145843, lms@inesc.pt

\section{Mattan Kamon}

Research Laboratory of Electronics, Dept. of Electrical Eng. and Comp. Science Massachusetts Institute of Technology

77 Massachusetts Ave., Room 36-881, Cambridge, MA 02139, USA, Tel: (617)-253-1205, Fax: (617)-258-7864, mattorle-vlsi.mit.edu

\begin{abstract}
In this paper we discuss generating low order models for efficient coupled circuitinterconnect simulation. The ever increasing speeds and shrinking feature sizes that are typical of state of the art integrated circuits designs have made coupling due to interconnect and packaging a very important, sometimes dominant, factor in system performance. The ability to efficiently perform coupled circuit-interconnect simulation before fabrication is essential in order to detect signal degradation due to delays or crosstalk. We first discuss methods of generating models for both two and three dimensional interconnect and then present a general, guaranteed-stable, model order reduction technique to reduce the order of the interconnect models.
\end{abstract}

Keywords

Interconnect, Modeling, Model Order Reduction, Coupled Simulation

\section{INTRODUCTION}

The dense interconnections and packaging structures used in compact electronic systems often produce electrical and magnetic interactions which interfere with system performance. With higher speeds, shrinking feature sizes and increasing complexity, the electrical and magnetic characteristics of such structures are becoming increasingly important factors in the behavior and performance of currently fabricated integrated circuits. Interconnect effects such as ringing, reflection, crosstalk, dispersion and attenuation may corrupt logic signals and prevent a circuit from functioning as specified. Such effects are difficult to simulate because they occur only as 
a result of an interaction between the field distribution in a complicated geometry of conductors, and the circuitry connected to those conductors.

In the design of communication, high-speed digital, and microwave electronic systems, long interconnect lines from printed circuit boards and packaging can also have an important impact on system performance. The electromagnetic fields produced by these lines can be approximated as two-dimensional and for this reason, including non-ideal transmission lines in circuit simulation has become a topic of much current research (Lin \& Kuh 1992, Celik \& Cangellaris 1996). In general, the behavior of stripline and microstrip printed circuit board traces, interchip connections on multi-chip modules, and coaxial cable connections are most easily represented by frequency-dependent scattering parameters. Since the scattering parameters may be derived from measured data, detailed finite-element simulation, or analytic formulas, a general approach to including transmission lines in circuit simulators is to allow for frequency-dependent elements specified by tables of data. The most straightforward approach to including general frequency-domain transmission line models in a circuit simulator is to calculate the associated impulse response using an inverse fast Fourier transform (Schutt-Aine \& Mittra 1988). Then, the response of the line at any given time can be determined by convolving the impulse response with an excitation waveform. Such an approach is too computationally expensive for use in general circuit simulation, as it requires that at every simulator timestep, the impulse response be convolved with the entire computed excitation waveform. An alternative approach is to approximate the frequency-domain representation with a rational function, in which case the associated convolution can be accelerated using a recursive algorithm (Lin \& Kuh 1992). Very efficient circuit simulation programs which handle RLCG transmission lines have been developed using such an approach, where the rational function approximation was derived using Padé or moment-matching methods (Bracken, Raghavan \& Rohrer 1992).

For three dimensional structures small compared to a wavelength, electromagnetic interactions between conductors can be represented arbitrarily accurately using a densely coupled resistor, inductor, and capacitor (RLC) network (Ruehli 1974). Although it is possible to simulate coupled circuit-interconnect problems by including this densely coupled RLC network with the transistor models in a circuit simulator, this can be a very inefficient approach. In fact, to reasonably accurately model complicated interconnect and packaging these $R L C$ models require a very large number of energy storage elements, and this will make subsequent circuit simulations using the models prohibitively expensive. Impulse response or recursive convolution techniques could be used, but more recently, model-order reduction schemes have been developed to reduce the number of these energy-storage elements without compromising the transient response properties of the model (Pillage \& Rohrer 1990, Bracken et al. 1992, Silveira, Kamon \& White 1995, Silveira, Kamon, Elfadel \& White 1996).

In this paper we discuss model order reduction via the Arnoldi process for efficient simulation of both two-dimensional and three-dimensional interconnect. In section 2 we will describe some of the techniques used in the accurate modeling 
of interconnect and packaging structures. Then, in section 3 we discuss commonly used techniques for performing model order reduction on the generated interconnect models and describe in some detail the Arnoldi-based model order reduction algorithm. Next, in section 4 we review the relevant issues that are involved in including interconnect models into standard circuit simulators such as SPICE or SPECTRE. In section 5 we describe an example that illustrates the techniques described. Finally, in section 6, we present conclusions and acknowledgments.

\section{INTERCONNECT AND PACKAGE MODELING}

In this section we describe some state-of-the art techniques available for generating models of two and three-dimensional interconnect amenable to model order reduction. We start in Section 2.1 by describing one approach for generating models from measurements, analytical models, or the output of an electromagnetic tool. Then in Section 2.2 we briefly describe the formulation used in a 3D electromagnetic modeling tool so that in Section 3 we can describe integrating model order reduction directly into an electromagnetic tool.

\subsection{Modeling Transmission Lines}

Efficient modeling of transmission lines involves fitting some appropriate matrix or input-output relationship to a rational function. The matrix or input-output relationship typically comes from measurement, an analytical model, or an electromagnetic tool which provides a scattering formulation, propagation factors, or admittance matrix over a range of frequencies. First, the ideal mode delays of the line are removed and then the rest of the propagation function is approximated by a rational function of an appropriate order. The mode delays can be returned later during simulation.

A numerically robust approach for computing a rational function approximation is to use frequency partitioning to reduce the dynamic range of the data. A set of local rational function approximations is first computed by linear fitting on each partitioned frequency interval. The set of all poles generated from the local approximations form an accurate set for a global rational function. A final global fitting can then be performed to adjust the residues of the approximation and to enforce correct steady-state computation on the model. Unfortunately the linear fitting of the local approximates is not guaranteed to produce stable poles. However, due to the partitioning, low order approximations are usually accurate enough in each section to avoid unstable poles. Moreover, unstable poles can normally be discarded without an impact on accuracy (Silveira, Elfadel, White, Chilukura \& Kundert 1994, Nguyen, Li \& Bai 1996).

Usually the construction of the global approximation from the local approximations introduces redundancies resulting in many more poles than necessary. It is therefore desirable to further reduce the order of the approximation. Reducing the 
order can be accomplished by first writing the system in state space form followed by the model order reduction techniques described in Section 3 (Silveira et al. 1994).

\subsection{Modeling 3D Coupling in Packaging Structures}

A wide range of integrated circuit and packaging design problems require accurate estimates of the coupling inductances of complicated three-dimensional structures. The frequencies of interest generally require magnetoquasistatic analysis, and a common approach is to apply finite-difference or finite-element techniques to a differential problem formulation. However, finite-element techniques require that the entire 3-D volume be discretized, and for complex structures, the generation of such a volume discretization can become cumbersome and require prohibitive execution time. Instead, volume-element methods can be applied to solving integral formulations of the problem in which case only the interior of the conductors need be discretized. Discretizing only the interior leads to many fewer unknowns than the finite-difference and finite-element techniques however the resulting system is dense which can be too expensive to solve by direct matrix factorization.

More recently methods for magnetoquasistatic analysis of complicated three-dimensional packages and interconnect have been proposed that allow the modeling of extremely complex packaging structures (Kamon, Tsuk \& White 1993). These methods use a standard volume-element discretization of an integral formulation from magnetoquasistatic analysis also known as the Partial Element Equivalent Circuit (PEEC) method (Ruehli 1979), but reformulate the discretized equations using mesh analysis. The mesh formulation leads to a dense system of equations which is solved iteratively using a rapidly converging Krylov-subspace method known as Generalized Minimal Residual (GMRES) (Saad \& Schultz 1986). Finally, since the system of equations is dense, the matrix-vector product required at each iteration of GMRES is expensive and to reduce its cost, the fast multipole method is used (Greengard \& Rokhlin 1987, Nabors \& White 1992). The combination of these techniques has been implemented in a packaging analysis program, named FASTHENRY, whose computational complexity and memory requirements grow linearly with the number of volume-elements required to discretize the conductors.

In FASTHENRY each conductor in a $t$-conductor system is approximated as piecewise-straight sections. To model skin and proximity effects, the volume of each straight section is then discretized into a collection of parallel thin filaments through which current is assumed to flow uniformly. By applying the Method of Moments to the magnetoquastatic equations, one can assign to each filament a resistance, inductance, and mutual inductance to every other filament in the problem.

A system of equations for the filament currents can be derived by assuming that the applied currents and voltages are sinusoidal, and that the system is in sinusoidal steady-state. Following the partial inductance approach in (Ruehli 1972), the filament current phasors can be related to filament voltage phasors by a branch impedance matrix, $Z=R+j \omega L$. 
The planar graph interconnecting the filaments creates a circuit and combining this formulation with Kirchhoff's voltage law via mesh analysis, the following equation relating the vector of mesh currents and the source branch voltages can be obtained

$M Z M^{T} I_{m}=V_{s}$

where $M \in \mathbb{R}^{m \times b}$ is the mesh matrix, $V_{s} \in \mathbb{C}^{m}$ is the mostly zero vector of source branch voltages and $I_{m} \in \mathbb{C}^{m}$ is the vector of mesh currents.

For a specific $\omega$, the complex admittance matrix which describes the external terminal behavior of a $t$-conductor system, denoted $Y_{t}=Z_{t}^{-1}$, can by derived from (1) by noting that $I_{t}=Y_{t} V_{t}$ where $I_{t}$ and $V_{t}$ are the terminal source currents and voltages of the $t$-conductor system. These values are related to the mesh quantities by $I_{t}=N^{T} I_{m}, V_{s}=N V_{t}$, where $N \in \mathbb{R}^{m \times t}$ is an easily constructed terminal incidence matrix. Hence, to compute the $i^{t h}$ column of $Y_{t}$, set $V_{t_{i}}=1$ and the rest of $V_{t_{i}}$ to zero. Solve (1) with a $V_{s}=N V_{t}$ and then extract the entries of $I_{m}$ associated with the source branches via $I_{t}=N^{T} I_{m}$.

One approach to coupling the above package models with circuits is to include a sparse tableau version of (1) in a circuit simulator instead of solving for the terminal behavior (Ruehli 1974). This approach has the drawback that the size of the system in (1) can easily be very large if high-accuracy is desired. Another possibility is to construct and solve (1) for various values of $\omega$ and then use the techniques described in 2.1 to compute a model for the package. However, a more computationally efficient approach is to form a state-space representation of (1),

$$
\begin{aligned}
\frac{d}{d t}\left(M L M^{T}\right) I_{m} & =-\left(M R M^{T}\right) I_{m}+N V_{t} \\
I_{t} & =N^{T} I_{m} .
\end{aligned}
$$

where $\boldsymbol{Z}$ has been expanded to $\boldsymbol{R}+j \omega \boldsymbol{L}$, and $j \omega$ replaced by $\frac{d}{d t}$. Then model order reduction techniques as described in the next section can be applied to derive a smaller approximation.

\section{MODEL ORDER REDUCTION}

Since the first papers on asymptotic waveform evaluation (AWE), Padé-based reduced order models have become standard for improving coupled circuit-interconnect simulation efficiency. A Padé approximation of $q^{\text {th }}$ order is defined as a rational function whose coefficients are selected to match the first $2 q-1$ moments of the transfer function of the system. For a SISO system given in state-space form

$$
\begin{aligned}
\boldsymbol{A} \dot{x} & =x+b u \\
y & =c^{T} x
\end{aligned}
$$


or in transfer function form $\boldsymbol{G}(s)=\boldsymbol{c}^{T}(\boldsymbol{I}-s \boldsymbol{A})^{-1} \boldsymbol{b}=\sum_{k=0}^{\infty} m_{k} s^{k}$, the moments are given as $m_{k}=\boldsymbol{c}^{T} \boldsymbol{A}^{k} \boldsymbol{b}$. Low order Padé approximates can be computed using direct evaluation of the moments, followed by a moment-matching procedure. In order to accurately compute higher order Padé approximates, it is necessary to use successive bi-orthogonalization combined with lookahead, as in the recent nonsymmetric Lanczos algorithms (Feldmann \& Freund 1995). Although nonsymmetric Lanczos methods plus lookahead can be used to generate Padé approximates of arbitrarily high order, there is no guarantee that a given approximate will be stable. It is therefore essential to postprocess the Padé approximate before using it in a circuit simulation program.

An alternative approach, which robustly generates a somewhat different approximation, can be derived using an Arnoldi process as in the GMRES algorithm. The idea behind this approach is similar to that of (Feldmann \& Freund 1995), and is that of selecting an orthonormal basis for the Krylov subspace $\mathcal{K}_{k}(\boldsymbol{A}, \boldsymbol{b})=\operatorname{span}\left\{\boldsymbol{b}, \boldsymbol{A b}, \boldsymbol{A}^{2} \boldsymbol{b}\right.$, $\left.\cdots, A^{k-1} b\right\}$. The orthogonality between the basis vectors makes the Arnoldi algorithm a better conditioned process than direct evaluation of the moments.

After $q$ steps, the Arnoldi algorithm returns a set of $q$ orthonormal vectors, as the columns of a matrix $V_{q} \in \mathbb{R}^{n \times q}$, and a $q \times q$ upper Hessenberg (tridiagonal plus upper triangular) matrix $H_{q}$. These two matrices are related by $V_{q}^{T} A V_{q}=H_{q}$. From this relation it can easily be shown that the moments of the transfer function can be written in terms of $\boldsymbol{H}_{q}$ and $\boldsymbol{V}_{q}$. Therefore the $q^{\text {th }}$ order Arnoldi-based approximation to $\boldsymbol{G}(s)$ can be written as

$G_{q}^{A}(s)=\|b\|_{2} c^{T} V_{q}\left(I-s H_{q}\right)^{-1} e_{1}$

corresponding to the state-space realization $\boldsymbol{A}_{q}=\boldsymbol{H}_{q}, \boldsymbol{b}_{q}=\boldsymbol{e}_{1}$, and $\boldsymbol{c}_{q}=\|\boldsymbol{b}\|_{2} \boldsymbol{V}_{q}^{T} \boldsymbol{c}$.

Block extensions to the Arnoldi approximation method, as well as the Lanczos methods, can be derived, which allow for direct computation of reduced-order models for interconnect or packaging structures with multiple inputs and outputs from the general MIMO system in (3).

For certain classes of RC, RL or LC circuits it has been shown that congruence transforms, like the Arnoldi algorithm, can generate guaranteed stable and passive reduced-order models. Recently a coordinate-transformed Arnoldi algorithm has been presented, that can generate arbitrarily accurate and guaranteed stable reducedorder models for RLC circuits. Efficient implementation of this algorithm requires simple modifications to the standard Arnoldi algorithm and its computational cost is roughly equivalent to that of PVL (Silveira et al. 1996).

Application of the Arnoldi technique to the 3D modeling problem is straightforward from a state-state formulation of the problem. For instance, for the 3D coupling problem in packaging structures described in Section 2.2, and from Eqn. (2) for the SISO case, the state-space formulation is readily obtained with $\boldsymbol{A}=-\left(\boldsymbol{M} \boldsymbol{R} \boldsymbol{M}^{T}\right)^{-1}$ $\left(M L M^{T}\right), b=\left(M R M^{T}\right)^{-1} N_{j}$ and $c=N_{i}$, where $N_{i}$ indicates the $i^{t h}$ column of $N$. 
Note that the computation of $b$ is inexpensive since $M R M^{T}$ is sparse. Also, because $L$ is dense, the dominant cost of each step of an Arnoldi process is a matrixvector product, $A \boldsymbol{x}=-\left(\boldsymbol{M} R \boldsymbol{M}^{T}\right)^{-1}\left(\boldsymbol{M L} \boldsymbol{M}^{T}\right) \boldsymbol{x}$. In practice, the matrix-vector cost dominates even when the dense part, $\left(M L M^{T}\right) x$, is rapidly computed with a hierarchical multipole-algorithm as in FASTHENRY.

A modified version of the standard Arnoldi algorithm which implements the coordinate-transformation described in (Silveira et al. 1996) can easily be derived. This algorithm can be shown to efficiently generate arbitrarily accurate and guaranteed stable reduced-order models for this problem.

\section{TIME-DOMAIN SIMULATION}

In order to account for the interconnect or package coupling effects when verifying the correctness of a design, it is necessary to include the interconnect model in the verification tool. Usually this implies that the generated model must be amenable to inclusion in a standard circuit simulation tool such as SPICE (Nagel 1975) or SPECTRE.

If the coupling effects are being obtained through some experimental procedure or from repeated usage of some detailed analytical tool that generates frequencydependent values, then the standard way to include this data into a simulator is via a convolution process at each timepoint. From the frequency-data, Laplace inversion or application of the inverse fast Fourier transform can be used to compute a corresponding impulse response which is then convolved with the excitation waveform. This approach is computationally demanding since at every simulator timepoint, the impulse response must be convolved with the entire computed excitation waveform. However, if some weak assumptions are made about the time-domain response, namely that it becomes "smooth" for large $t$, then the cost of computing the convolution integral can be significantly reduced (Kapur, Long \& Roychowdhury 1996).

If the frequency-domain data is approximated by a rational function as described in Section 2.1 then a recursive algorithm can be used which reduces the cost of computing the convolution integral at each timepoint to a constant factor (Lin \& Kuh 1992).

A different approach can be applied if the interconnect model is in a state-space form. In this case it is possible to directly include the model into the simulator, given that the model itself is simply a set of ordinary differential equations, similar to the circuit equations themselves. Therefore one can simply enlarge the list of variables that the simulator will solve for by adding to it the state vector. Given that the computational cost of circuit simulation grows superlinearly with the number of unknowns, it is important that the interconnect model be as small as possible.

Of course if the generated model is realizable, which is not always the case, then it is also possible to synthesize an RLC circuit from the state equations. In this case no modifications need to be introduced to the simulator and the model can be can be used directly. 


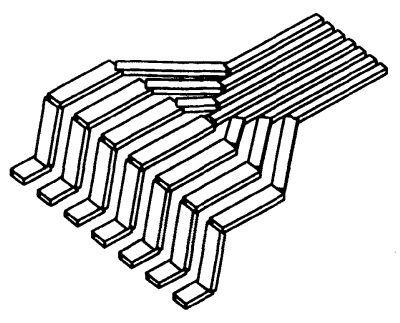

(a)

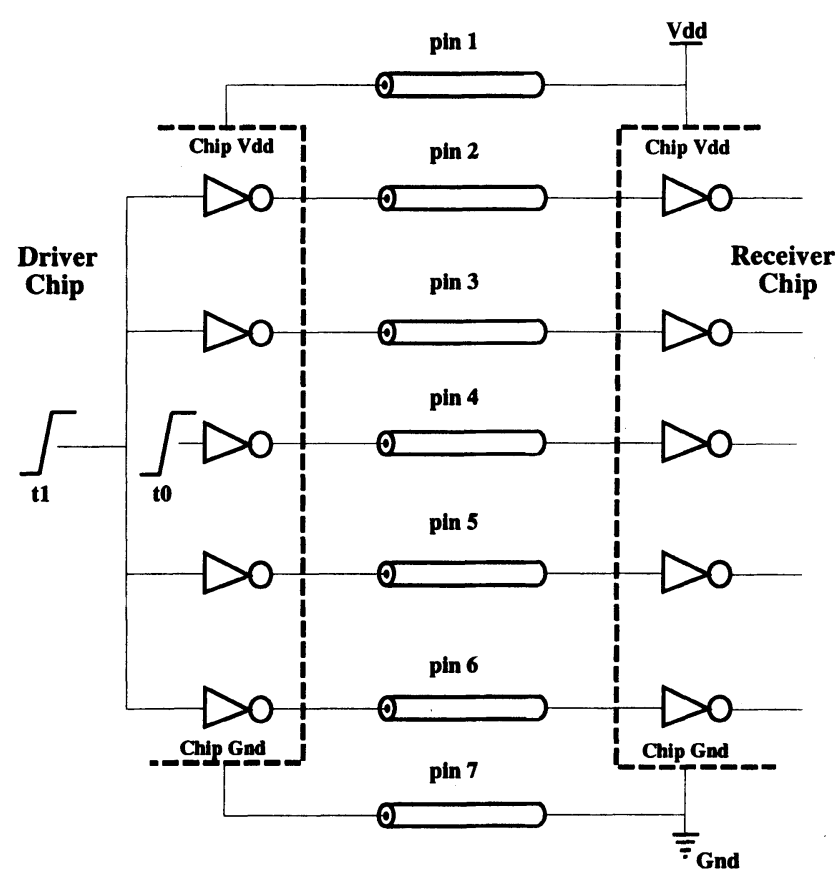

(b)

Figure 1 (a) Seven pins of a cerquad pin package. (b) General configuration for the connection between receiver and driver chips. All the circuit elements inside the same chip share that chip's power and ground.

\section{EXPERIMENTAL RESULTS}

In the preceding sections, we described algorithms to compute reduced order models of interconnect and packaging structures using either Padé or Arnoldi-based algorithms. In this section we show how such models can be used for coupled circuitinterconnect simulation. First the accuracy of the approximations is examined when used to obtain reduced-order models for the frequency-dependent admittance for a small set of package pins. The model generated for the packaging strcuture is used in a coupled circuit interconnect simulation that shows how crosstalk between the pins of the package can affect the performance and integrity of the signals.

Consider the small set of package pins. as shown in Figure 1-a). To compute the resistance and inductance matrices with FASTHENRY, the pins were discretized into three filaments along their height and four along their width producing a system of size $m=887$. This allows modeling of changes in resistance and inductance due to skin and proximity effects. The model to be produced for this seven terminal system has seven inputs and seven outputs. 

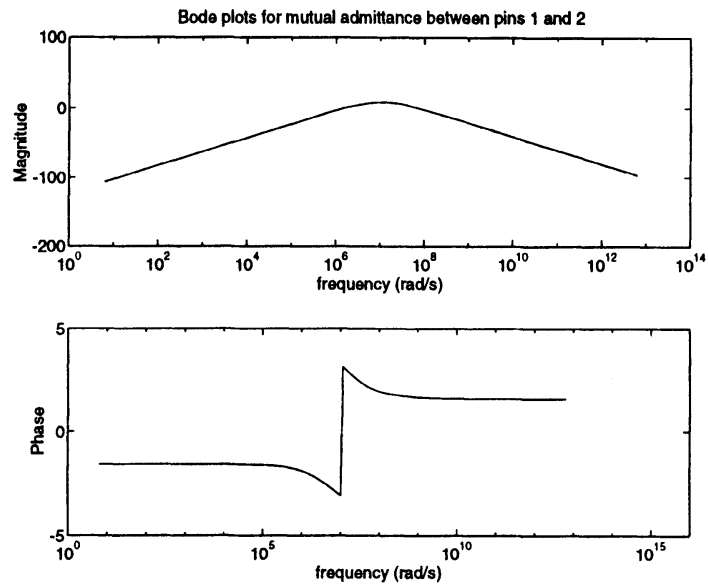

Figure 2 Bode plot for an Arnoldi approximation to the coupled admittance transfer function between pins 1 and 2 . The curves are indistinguishable from the exact solution which is also plotted.

\subsection{Accuracy Comparisons}

Figure 2 shows the Bode plot of an $8^{\text {th }}$ order Arnoldi-derived approximations to the coupled admittance transfer function between pins 1 and 2. Also shown in the picture is the exact admittance transfer function obtained by eigendecomposition of the full system. As can be seen from the plot, the approximated model is virtually indistinguishable from the full transfer function. This same accuracy could be obtained by constructing an approximant from the frequency data-points, as described in Section 2.1 (Silveira et al. 1995). However this latter technique would be extremely inefficient in this situation, since if if discrete frequency data were generated, generating 50 frequency points would require roughly 30 times the number of Arnoldi iterations needed by the direct state-space approach described in Section 2.2.

\subsection{Coupled Simulation Results}

To investigate the crosstalk effects between the package pins in Fig 1-a), the configuration shown in Fig. 1-b) is used where it was assumed that the five middle lines carry output signals from the chip and the two outer pins carry power and ground. The signals are driven and received with CMOS inverters which are capable of driving a large current to compensate for the impedance of the package pins. The capacitance is assumed to be $8 \mathrm{pF}$ and the interconnect from the end of pin to the receiver is modelled with a capacitance of $5 \mathrm{pF}$. A $0.1 \mu F$ decoupling capacitor is connected between the driver's power and ground to minimize supply fluctuations. The frequency dependence of each element in the admittance matrix is modeled via Arnoldi-based approximations of $8^{\text {th }}$ order. These models are then incorporated into SPICE 3 as a 

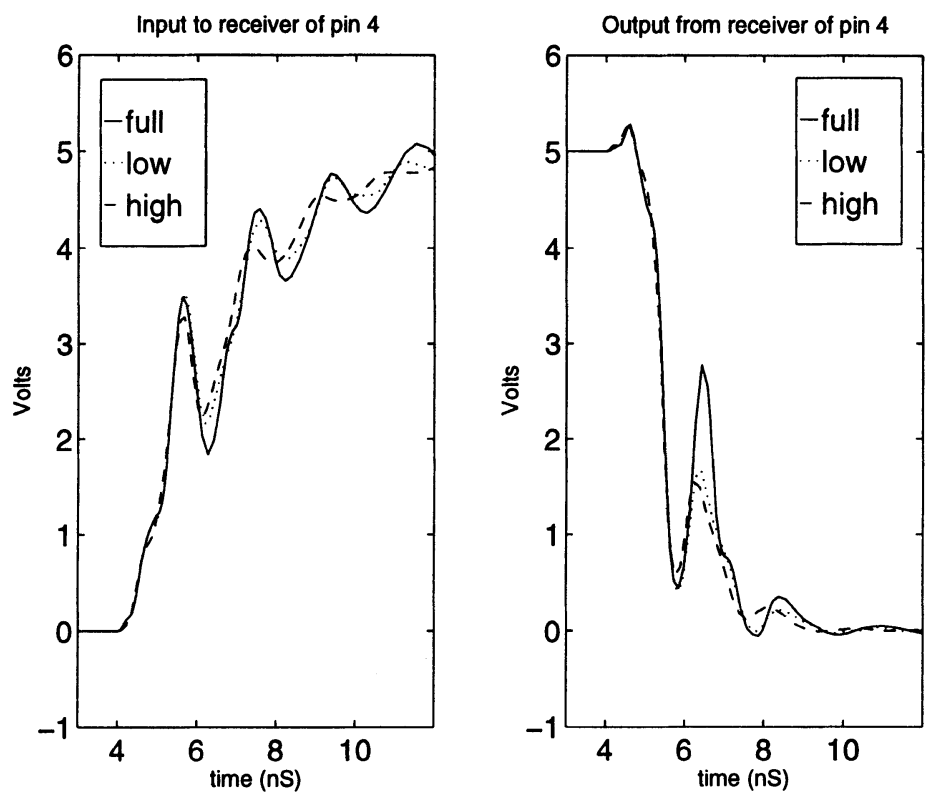

Figure 3 Results of the timing simulation for the output of the receiver gate connected to pin 4 when the adjacent pins switch 1 ns after pin 4 . Comparison of the waveforms on Pin 4's receiver using a model constructed from frequency dependent data (full), a constant value from low frequency (low), and a constant value from high frequency (high).

frequency-dependent voltage-controlled current source vCCS. As a sample time domain simulation, imagine that at time $t_{0}=4 \mathrm{~ns}$ the signal on pin 4 of Fig.1-b) is to switch from high to low and pins $2,3,5$, and 6 are to switch from low to high but that due to delay on chip, pins $2,3,5$, and 6 switch at $t_{1}=5 \mathrm{~ns}$. In this case, significant current will suddenly pass through the late pins while pin 4 is in transition. Due to crosstalk, this large transient of current has significant effects on the input of the receiver on pin 4, as shown in the solid line in Fig. 3. Note that the input does not rise monotonically. The curve also also shows that the bump in the waveform is carried through to the output of receiver, as a large glitch.

As a demonstration of the importance of frequency dependent analysis, Fig. 3 also shows the waveforms resulting from simulations using constant resistance and inductance values corresponding to the high or low frequency limits (the dashed and dotted waveforms respectively). Note that for the receiver input waveforms, the large voltage bump swings by approximately $0.5 \mathrm{~V}$ more for the full frequency-dependent case. While this is small on the input, this is a very sensitive region for the receiver and doubles the size of the output glitch. 


\section{CONCLUSIONS AND ACKOWLEDGEMENTS}

In this paper we reviewed some of the commonly used techniques for generating accurate models for interconnect and packaging coupling, that are amenable to efficient circuit level simulation. As an example of the capabilities and effectiveness of such techniques, an approach for computing reduced-order models of magnetoquasistatic coupling in complicated 3-D structures was reviewed. This approach shows how the combination of an appropriate formulation of the problem with the use of robust numerical techniques can lead to models that can be used to produce results within acceptable timeframes without compromising accuracy. Numerical examples were presented that show how accurate reduced-order models can be generated and used in coupled circuit-interconnect simulation.

The authors wish to acknowledge the very helpful discussions with Dr. Ibrahim Elfadel of IBM T.J. Watson, and Prof. Jacob White of MIT. This work was partially supported by the Defense Advanced Research Projects Agency, the National Science Foundation and the Portuguese JNICT programs PRAXIS XXI and FEDER under contracts 2/2.1/T.I.T/1661/95 and 2/2.1/T.I.T/1639/95.

\section{REFERENCES}

Bracken, J. E., Raghavan, V. \& Rohrer, R. A. (1992). Interconnect Simulation with Asymptotic Waveform Evaluation, IEEE Trans. Circuits Syst. 39(11): 869-878.

Celik, M. \& Cangellaris, A. C. (1996). A general dispersive multiconductor transmission line model for interconnect simulation in spice, International Conference on Computer Aided-Design, San Jose, California, pp. 563-568.

Feldmann, P. \& Freund, R. W. (1995). Efficient linear circuit analysis by Padé approximation via the Lanczos process, IEEE Iransactions on Computer-Aided Design of Integrated Circuits and Systems 14: 639-649.

Greengard, L. \& Rokhlin, V. (1987). A fast algorithm for particle simulations, Journal of Computational Physics 73(2): 325-348.

Kamon, M., Tsuk, M. J. \& White, J. (1993). Fasthenry, a multipole-accelerated 3-d inductance extraction program, Proceedings of the ACM/IEEE Design Automation Conf., Dallas.

Kapur, S., Long, D. E. \& Roychowdhury, J. (1996). Efficient time-domain simulation of frequency-dependent elements, International Conference on Computer Aided-Design, San Jose, California, pp. 569-573.

Lin, S. \& Kuh, E. S. (1992). Transient Simulation of Lossy Interconnects Based on the Recursive Convolution Formulation, IEEE Trans. Circuits Syst. 39(11): 879-892.

Nabors, K. \& White, J. (1992). Fast capacitance extraction of general three-dimensional structures, IEEE Trans. on Microwave Theory and Techniques.

Nagel, L. W. (1975). SPICE2: A Computer Program to Simulate Semiconductor Circuits, Technical Report ERL M520, Electronics Research Laboratory Report, University of California, Berkeley, Berkeley, California.

Nguyen, T. V., Li, J. \& Bai, Z. (1996). Dispersive coupled transmission line simulation using an adaptive block lanczos algorithm, International Custom Integrated Circuits Conference, pp. 457-460.

Pillage, L. T. \& Rohrer, R. A. (1990). Asymptotic Waveform Evaluation for Timing Analysis, 
IEEE Trans. CAD 9(4): 352-366.

Ruehli, A. E. (1972). Inductance calculations in a complex integrated circuit environment, IBM J. Res. Develop. 16: 470-481.

Ruehli, A. E. (1974). Equivalent Circuit Models for Three-Dimensional Multiconductor Systems, IEEE Trans. on Microwave Theory and Techniques MTT-22(3): 216-221.

Ruehli, A. E. (1979). Survey of computer-aided electrical analysis of integrated circuit interconnections, IBM J. Res. Develop. 23(6): 626-639.

Saad, Y. \& Schultz, M. H. (1986). GMRES: A generalized minimal residual algorithm for solving nonsymmetric linear systems, SIAM Journal on Scientific and Statistical Computing 7: 856-869.

Schutt-Aine, J. E. \& Mittra, R. (1988). Scattering Parameter Transient Analysis of Transmissions Lines loaded with Nonlinear Terminations, IEEE Trans. on Microwave Theory and Techniques MTT-36: 529-536.

Silveira, L. M., Elfadel, I. M., White, J. K., Chilukura, M. \& Kundert, K. S. (1994). Efficient Frequency-Domain Modeling and Circuit Simulation of Transmission Lines, IEEE Trans. on Components, Packaging, and Manufacturing Technology - Part B: Advanced Packaging 17(4): 505-513.

Silveira, L. M., Kamon, M., Elfadel, I. \& White, J. K. (1996). A coordinate-transformed Arnoldi algorithm for generating guaranteed stable reduced-order models of rlc circuits, International Conference on Computer Aided-Design, San Jose, California, pp. 288-294.

Silveira, L. M., Kamon, M. \& White, J. K. (1995). Algorithms for Coupled Transient Simulation of Circuits and Complicated 3-D Packaging, IEEE Trans. on Components, Packaging, and Manufacturing Technology, Part B: Adv. Packaging 18(1): 92-98.

\section{BIOGRAPHIES}

Luís Miguel Silveira was born in Lisbon, Portugal. He received the Engineer's (summa cum laude) and Master's degrees in Electrical and Computer Engineering in 1986 and 1989, from Instituto Superior Técnico (IST) in Lisbon, and the M.S., E.E. and Ph.D. degrees in 1990, 1991 and 1994 from the Massachusetts Institute of Technology, Cambridge, MA. Mr. Silveira is currently an associate professor at IST, a reseacher in the Electronics division of INESC, Instituto de Engenharia de Sistemas e Computadores, and a member of the Lisbon Center of the Cadence European Laboratories. His research interests are in various aspects of computer-aided design of integrated circuits with emphasis on parallel computer algorithms and the theoretical and practical issues concerning numerical simulation methods for circuit design problems. Mr. Silveira is a member of the IEEE and Sigma Xi.

Mattan Kamon received the B.S degree in engineering science from the Pennsylvania State University in 1991, the M.A. degree in mathematics also from the Pennsylvania State University in 1991, and the S.M. degree in electrical engineering and computer science from the Massachusetts Institute of Technology in 1994. $\mathrm{He}$ is presently working towards the Ph.D degree at the Massachusetts Institute of Technology. 